\title{
Using a behavior analytics approach to change nursing care of extremely premature infants: A pilot study
}

\author{
Jenn Gonya*1, Jessica Niski ${ }^{2}$, Nicole Cistone ${ }^{3}$ \\ ${ }^{1}$ Center for Perinatal Research, The Research Institute at Nationwide Children's Hospital, Columbus, Ohio, United States \\ ${ }^{2}$ Dept. of Physical Therapy, School of Pharmacy \& Health Professions, Creighton University, Omaha, Nebraska, United States \\ ${ }^{3}$ Nationwide Children's Hospital Neonatal Intensive Care Unit Small Baby Program, Columbus, Ohio, United States
}

Received: November 2, 2018

DOI: $10.5430 /$ jnep.v9n5p14
Accepted: December 17, $2018 \quad$ Online Published: December 30, 2018

URL: https://doi.org/10.5430/jnep.v9n5p14

\begin{abstract}
The neonatal intensive care unit (NICU) is, inherently, a trauma environment for the extremely premature infant. This trauma is often exacerbated by nurse caregiving practices that can be modified and still remain effective. Our study explored how behavior analytics could be used to implement an intervention known as Care by Cues and how the intervention might, ultimately, impact infant physiologic stability.
\end{abstract}

Key Words: Trauma, Neonatal intensive care unit, Infant, Behavior analytics approach, Care by Cues

\section{INTRODUCTION}

The neonatal intensive care unit (NICU) environment is a source of continual infant dysregulation. Daily stressors such as ventilation, intravenous lines, nurse caregiving, etc. challenge the behavioral and physiologic capacities of extremely premature infants and impact their long-term developmental outcomes. ${ }^{[1-3]}$ This occurs to such an extent that the NICU is often described as a trauma environment ${ }^{[4]}$ and there has been an increasing call for changing NICU practice. In response, numerous neonatal professional associations have jointly issued best-practice standards ${ }^{[5]}$ that outline five key areas for advancing NICU care: protection of sleep, management and assessment of stress and pain, provision of age-appropriate caregiving, support of family-centered care, and promotion of a healing environment.

Although not explicitly stated, the fundamental component that connects each of the five key areas is infant regulation. Extremely premature infants miss their last trimester of development in an intrauterine environment that naturally sup- ports regulation through maternal responsiveness to infant activity, sound buffering, tucked positioning with responsive boundaries, buoyant environment, temperature regulation and nutrition. Consequently, in the extrauterine environment, extremely preterm infants require external supports to promote regulation and the development of independent regulatory capacity over time. ${ }^{[6]}$ Through caregiving that is co-regulatory, that is purposefully aimed at maintaining regulated infant behavior and physiology, extremely premature infants are able to manage and cope with their environments. Extremely premature infants who are regulated are able to 1) make smooth transitions between sleep and awake states, 2) perform self-calming behaviors (e.g., bringing hands together, grasping a finger, pacing respiration, etc.) in response to stress or pain, and 3) maintain a quiet, alert state for a period of time. ${ }^{[7]}$ These activities support an infant's availability for meaningful human interaction and are critical for appropriate development. ${ }^{[8]}$

Through this lens, the goal of nurse caregiving becomes one

*Correspondence: Jenn Gonya; Email: jenn.gonya@ nationwidechildrens.org; Address: Center for Perinatal Research, The Research Institute at Nationwide Children's Hospital, Columbus, Ohio, United States. 
of supporting infant regulation through co-regulatory activities, rather than completing a set of given care tasks. It is then imperative to investigate ways in which nurses can be effectively taught how to co-regulate extremely premature infants in the NICU during routine caregiving.

\section{Research questions}

The primary purpose of this pilot study was to investigate the effect of an intervention, known as Care by Cues, on nurse caregiving knowledge and routine caregiving practice in the NICU. The secondary aim was to explore the potential impact of the Care by Cues intervention on infant behavior and physiology.

\section{Methodology}

\subsection{The participants}

A convenience sample of six nurses was recruited based on their responses to an open call for participants at a quarterly departmental meeting. Nurses were eligible to participate in the study if they had worked in the specialized extreme prematurity unit for at least five years and had consistently scheduled shifts during the intervention time period. To address the second aim, one infant from the unit who met inclusion criteria was recruited (i.e., was extremely premature, was admitted to the specialized unit, was 28 weeks corrected age, was a non-surgical patient, and did not have congenital anomalies). The study was approved by the Institutional Review Board with appropriate consent policies for human subjects research.

\subsection{The intervention (Care by Cues)}

Although routine caregiving of extremely premature infants can be perceived as simplistic and task focused in form (e.g., counting respirations, changing the diaper, placing the feed on the pump, etc.), it is actually quite complex and dynamic. What occurs before, during, and after routine caregiving contributes to each infant's overall regulatory state. ${ }^{[9]}$ Therefore, we performed a task analysis for each phase of care (i.e., before care, during care, and after care) using a behavioral analytic approach. ${ }^{[10]}$ Learning and behavioral objectives were then developed, and pedagogical methodologies were designed to address each objective. Each phase of routine caregiving was then taught in a forward chain with specified levels of mastery. Figure 1 outlines the behavioral learning sequence.

The intervention began with each nurse receiving a Care by Cues folder that consisted of educational resources such as evidence based articles discussing the effects of handling preterm newborns during caregiving, NICU environmental factors, neuroprotective developmental care, sleep and brain development, individualized developmental care, awareness of preterm infants' behavioral cues, the Care by Cues Algorithm (see Figure 2), and a table that defined and described infant sleep-awake states and stress signs.

This was followed with a direct instruction session that was structured to provide education to the six nurses who were recruited for the pilot study. The 50-minute, continued education session utilized a PowerPoint format to provide insight and education on routine NICU caregiving. The learning objectives were to describe the short and long-term adverse consequences of sleep disturbances and stressors of routine NICU care, explain the sleep requirements of infants, and review the signs of stress and self-regulating behaviors of infants.

During the presentation, two previously recorded educational videos (3-9 minutes in length) were shown to reflect current caregiving interactions in the NICU. These videos were used to provide insight into a caregiving interaction between the nurse and the patient. Following review of the videos, the nurses were asked to discuss their observations. Facilitator use of questioning strategies initiated open discussion through questions such as, "Was the infant asleep or awake prior to the initiation of care?" "What did you observe?" "What task(s) were absolutely necessary during this care?" and "What stress signs did the infant exhibit?"

\begin{tabular}{|c|c|c|}
\hline Phase A: Initiation of Care & Phase B: Care by Cues & Phase C: Transition to Sleep \\
\hline $\begin{array}{l}\text { A1: Maintenance of proximity } \\
\text { to the infant with observation } \\
\text { without alarm prompting or } \\
\text { cry prompting }\end{array}$ & $\begin{array}{l}\text { B1: Identification of infant dysregulation cues } \\
\text { (non-alarm): } \\
\text { - Limb Extension/Flexion } \\
\text { - Whole Body Movement } \\
\text { - Facial Movement } \\
\text { - Eye Movement }\end{array}$ & $\begin{array}{l}\text { C1: Identification of infant cues for transitioning to a } \\
\text { sleep state: } \\
\text { - } \quad \text { Breathing regularity } \\
\text { - Whole Body Movement } \\
\text { - Sucking } \\
\text { - } \quad \text { Eye }\end{array}$ \\
\hline $\begin{array}{l}\text { A2: Identification of an infant } \\
\text { awake state }\end{array}$ & $\begin{array}{l}\text { B2: Identification of antecedent condition of } \\
\text { the dysregulation cue }\end{array}$ & $\begin{array}{l}\text { C2: Maintenance of proximity to the infant with } \\
\text { observation without alarm prompting or cry prompting }\end{array}$ \\
\hline $\begin{array}{l}\text { A3: Initiation of care with an } \\
\text { intentional introduction }\end{array}$ & $\begin{array}{l}\text { B3: Implementation of accurate consequent } \\
\text { condition of the dysregulation cue }\end{array}$ & $\begin{array}{l}\text { C3: Implementation of accurate consequent condition } \\
\text { of dysregulation cue }\end{array}$ \\
\hline & $\begin{array}{l}\text { B4: Evaluation of infant response to the } \\
\text { implemented consequent condition }\end{array}$ & $\begin{array}{l}\text { C4: Evaluation of infant response to the implemented } \\
\text { consequent condition }\end{array}$ \\
\hline
\end{tabular}

Figure 1. Behavioral learning sequence for the Care by Cues nurse routine caregiving intervention. Participants worked from top to bottom within each Phase and then forward from Phase A to Phase C 


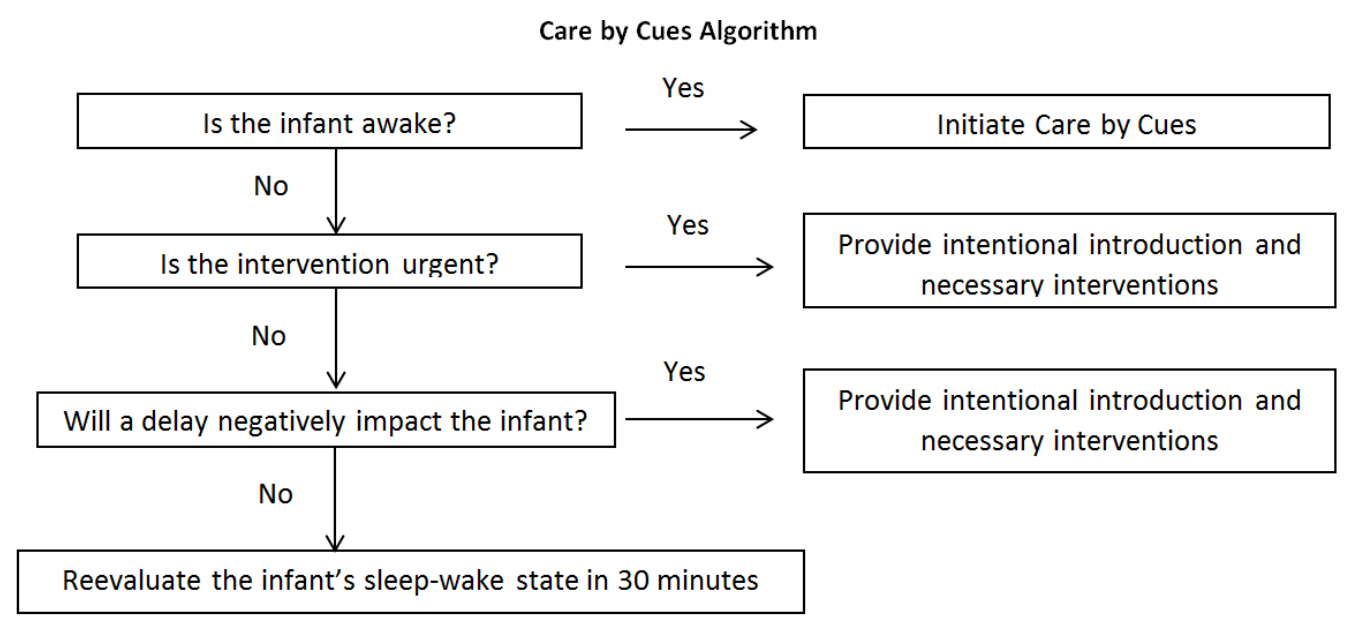

Figure 2. Adapted algorithm presented to nurses in the intervention to aid their clinical decision-making on whether or not to initiate routine care

Two weeks after initial education, participants received the first assignment packet, which focused on Phase A. This was subsequently followed with Phase $\mathrm{B}$ and Phase $\mathrm{C}$ assignment packets, two and four weeks later, respectively. Each set of assignments assessed knowledge and provided scaffolding for learning and reflection.

During Phase A (Focus: What Happens Before Routine Care Starts?), participants were asked to write the definition of Care by Cues in their own words. They were also instructed to read a journal article about infant sleep and awake states and to review an adapted algorithm ${ }^{[11]}$ created for the project to support decision-making when determining whether or not a caregiving interaction should be initiated (see Figure 2).

Additionally, participants had to perform an online clinical check-in to describe what they observed about their patient's awake state. The final assignment involved completing two Intentional Observation sheets in which they rated the difficulty of implementing an Intentional Introduction (i.e., placing hands on the infant to provide gentle containment and counting for at least 5 seconds before performing any routine caregiving tasks) and described what they observed during the implementation of this intervention. Instructors were available for support and an electronic, web-based groupchat was formed as a resource to answer questions and to provide feedback to participants.

Built into Phase A, was a preliminary assignment for Phase $\mathrm{B}$ that introduced nurses to the process of critically analyzing a routine care session. Nurses were provided a flash drive containing a video of a routine caregiving session performed by an unidentified nurse in another unit. Participants were asked to view the video twice. During the first viewing, participants were asked to focus on identifying infant stress behaviors. In the second viewing, participants selected five of the previously identified stress behaviors and were asked to focus on when during the interaction the stress behavior occurred (e.g., at the onset of care, during a diaper change, when there was a loud noise, etc.). The goal of the exercise was to begin to conceptually connect infant stress behaviors with antecedent conditions.

In Phase B (Focus: What Happens During Care?), participants were again asked to write the definition of Care by Cues in their own words. Participants then reviewed a summary sheet of infant stress signs, described the stress signs they were observing in their patient through the online Clinical Check-In, and completed two Intentional Observation sheets. In this phase, nurses were videotaped during one of their own routine caregiving sessions. Following taping, participants met with instructors to analyze the video together. During the analysis, nurses viewed their personal video twice. On the first viewing, they were asked to identify their patient's stress behaviors and score their regulation using a rubric adapted from the Assessment of Preterm Infant Behavior (APIB) ${ }^{[12]}$ In the second viewing, participants selected five stress behaviors and identified both antecedent and, additionally, consequent conditions of each stress behavior.

In the last phase, Phase C (Focus: What Happens After Care?), nurses completed their final handwritten definition of Care by Cues. During this phase, participants were asked to practice performing Intentional Exits (i.e., placing hands on the infant to provide gentle containment and counting for at least 5 seconds at the end of care before leaving the bedside). Finally, nurses also obtained a second video of themselves during care and performed analysis using the clinical checkin to describe what they noticed about their patients during or after the Intentional Exits. 


\subsection{The design}

Mixed methods were used for the study with qualitative and quantitative components including a multiple baseline single case approach. Data with regard to nursing knowledge and routine caregiving practice were collected at baseline, during two-week intervals, and post-intervention. Infant behavioral and physiologic data were collected continuously for a 48-hour time period containing randomized segments of intervention and control conditions.

\subsection{The measures}

\subsubsection{Nursing knowledge of Care by Cues}

Nursing responses to the three assignments for defining Care by Cues were scored using a three-point rubric (see Table
1) with whole- and half-value credit possible. Participants scored a 1 if they provided an Externalized Definition (i.e., textbook, global, or general definition); a 2 for an Internalized - Unidirectional Definition (i.e., unidirectional wording, identification of infants as participants in the care); and a 3 for an Internalized-Bi-Directional Definition (i.e., identified care as a transactional process with infant and nurse providing actions and responses). Half credit point values were assigned for responses that included equal percentages of wording from two categories. In addition, nursing knowledge was measured through the Phase A video analysis worksheet and completion of pre- and post-intervention surveys that included questions about infant stress cues. Questions were scored and percentage correct values were computed.

Table 1. Scoring rubric for assessing nurses' knowledge of Care by Cues

\begin{tabular}{|c|c|c|c|c|}
\hline 1 & 1.5 & 2 & 2.5 & 3 \\
\hline $\begin{array}{l}\text { Externalized Definition } \\
\text { (e.g., textbook, global, } \\
\text { general) }\end{array}$ & $\begin{array}{l}\text { Elements of both } \\
\text { externalized and } \\
\text { internalized- } \\
\text { unidirectional }\end{array}$ & $\begin{array}{l}\text { Internalized Definition } \\
\text { - Unidirectional } \\
\text { (identified participants in the } \\
\text { care, but viewed care as } \\
\text { occurring in one direction) }\end{array}$ & $\begin{array}{l}\text { Elements of both } \\
\text { internalized- } \\
\text { unidirectional and } \\
\text { internalized- } \\
\text { bi-directional }\end{array}$ & $\begin{array}{l}\text { Internalized Definition } \\
\text { - Bi-directional (identified } \\
\text { participants in the care as } \\
\text { transactional with both participants } \\
\text { providing cues and responses }\end{array}$ \\
\hline Wording Examples & & Wording Examples & & Wording Examples \\
\hline $\begin{array}{l}\text { - Developmentally-friendly } \\
\text { - Neurologically protective } \\
\text { - State of tolerance } \\
\text { - Optimize } \\
\text { neurodevelopment } \\
\text { - Improve outcomes }\end{array}$ & & $\begin{array}{l}\text { - Patient-led } \\
\text { - Infant-centered } \\
\text { - Best suited to the infant } \\
\text { - Cueing } \\
\text { - Infant sleep-awake states } \\
\text { - Positive stimulation }\end{array}$ & & $\begin{array}{l}\text { - Engaging } \\
\text { - Meaningful human interaction } \\
\text { - Knowing when to start and stop } \\
\text { - Intentional } \\
\text { - Modifying care }\end{array}$ \\
\hline
\end{tabular}

2.4.2 Nursing practice-Routine caregiving with Care by Cues

Intervention implementation and nursing identification of caregiving effects were assessed through four primary methods: Intentional Introduction and Exit Observation Sheets (two submissions for each), Clinical Check-In assignments (one submission for each teaching phase), self-video analysis (one video in each teaching phase of $\mathrm{B}$ and $\mathrm{C}$ ), and the Clinical Mentorship Feedback Form (one submission in each teaching phase of B and C). In the Intentional Introduction and Exit Observation sheets, nurses identified whether or not they were able to implement an Intentional Introduction or Exit and rated how difficult the implementation was. There was also a section of open-ended questions asking nurses to describe what they observed during these time periods. In each teaching phase, Clinical Check-In assignments involved nurses in posting, in a private e-mail group, what they were "seeing" and "experiencing" with their patients during the Care by Cues intervention.

During Phases B and C, nurses were asked to arrange with instructors for videotaping of one of their routine caregiving sessions. The video was then analyzed by the participant with one of the intervention instructors. Using a worksheet

Published by Sciedu Press as a framework, the nurse and instructor observed infant behavior, identified infant stress cues, and then discussed the antecedent and consequent conditions of the behavior. After video analysis, nurses worked with instructors to complete the Clinical Mentorship Feedback Form that documented identified strengths, areas for growth, and level of Care by Cues proficiency. Proficiency levels were rated on a scale from 1-4 with 1 being "Performs with extensive guidance" and 4 being "Performs with proficiency."

Once nurse-instructor video analysis was complete, video was sent to the blinded research team to analyze structural components of routine caregiving with care by cues. Actual implementation of the Intentional Introduction and the Intentional Exit were validated and caregiving variables (i.e., amount of care time, number of caregiving tasks, type of caregiving tasks) before, during, and after care were quantified.

\subsubsection{Nursing evaluation of the Care by Cues process}

A 9-question survey was administered to nurses after completion of the Care by Cues training. Responses were scored along a five-point Likert scale from 1 (Strongly Agree) to 5 (Strongly Disagree) and addressed questions such as "I 
am confident in my understanding of what Care by Cues means" to "I am confident in my critical thinking skills and use of visual assessment to determine infant stability." Additionally, two open-ended questions asked nurses to identify a) barriers in larger scale implementation of Care by Cues and b) additional Care by Cues education they would like to see in the future.

\subsection{Infant behavioral and physiologic effects of the in- troduction of care (IOC) segment of Care by Cues}

Infant regulation, dysregulation and physiologic variability were assessed through a single case multiple baseline intervention/withdrawal design. Observations were made through real time review of video recording with data collected regarding infant behaviors, heart rate, and oxygen saturation. One infant was selected for analysis based on availability during the Care by Cues intervention time period and fulfillment of inclusion criteria. Continual video recording of the infant occurred for a 48-hour time period during which the IOC segment of the Care by Cues intervention was randomized between IOC and withdrawal. Cameras were set up to only view the infant and the hands of the caregiver providing care/interaction. No identifying information of family members, nurses, or other caregivers were recorded.

After recording was complete, the blinded research team reviewed the video and collected continuous data points starting 5 minutes before routine care, continuing throughout the caregiving activities, and for 10 minutes following completion of routine caregiving activities. Infant regulation/dysregulation behaviors were defined, identified, and tallied based on methods adapted from the Newborn Individualized Developmental Care and Assessment Program (NIDCAP) protocol. ${ }^{[13]}$ Infant behaviors of focus included: extremity extension, finger splay, squirming, loss of tone, stop signs, sitting on air, grasping, tucking, mouthing, nonnutritive sucking (NNS), and eye opening. These behaviors were selected from the NIDCAP observation sheet given the ability to readily identify these behaviors in real time review of video recordings. Extremity extension, finger splay, squirming, loss of tone, stop signs, and sitting on air were selected to represent infant stress or dysregulation while grasping, tucking, mouthing, NNS, and eye opening were selected as representative behaviors of infant regulation. Behaviors were tallied separately for stress/dysregulation behaviors and regulation behaviors during each identified epoch of time ( 5 minutes before routine care, throughout the care giving activities and during the 10 minutes following completion of routine cares). Behavioral data were collected for the 16 total care giving interactions recorded in the 48 hour period. Physiologic data (i.e., heart rate and oxygen saturation) were also collected at 10-second intervals and plotted over time to identify trends before, during, and after routine caregiving. Physiologic data were recorded for one ABAAB, baseline(A)/intervention(B)/withdrawal(A) cycle within the 48-hour period. ${ }^{[14]}$

\subsection{Data analysis}

Quantitative data was analyzed using the Statistical Package for the Social Sciences (SPSS) version 25 and Microsoft Excel 2016. Descriptive statistics were used to describe nursing observations during the IOC and Intentional Exit segments of Care by Cues, survey responses, and routine caregiving (e.g., length of care time and Care by Cues segments, the number of nursing tasks during care, etc.). Traditional behavior analytic line graphs ${ }^{[10]}$ were used to detect patterns and trends in nursing behavior and infant behavioral and physiologic responses.

\section{RESULTS}

\subsection{Nursing knowledge of Care by Cues}

Nurses' written responses advanced over the three phases of instruction (see Figure 1) with all nurses scoring at least a 2 (Internalized Definition) by the final phase (see Table 2). One nurse achieved the top score by Phase III, with two additional nurses scoring 2.5. Pre- and post- survey scores indicated that by Phase III, all nurses could answer questions about Care by Cues with at least $85 \%$ accuracy.

Table 2. Rubric scores for each of the six nurses across all three phases of instruction

\begin{tabular}{llll}
\hline Nurse & Phase I & Phase II & Phase III \\
\hline 1 & 1 & 2 & - \\
2 & 1 & 2 & 3 \\
3 & 2 & 2 & 2.5 \\
4 & 1.5 & - & 2 \\
5 & 2 & 2 & 2 \\
6 & 2.5 & 2.5 & 2.5 \\
\hline
\end{tabular}

\subsection{Nursing practice-Routine caregiving with Care by Cues}

For the Intentional Introduction observations, 100\% (11/11) of submissions indicated that nurses felt that they were able to perform an Intentional Introduction for the full amount of required time with $91 \%(10 / 11)$ of nurses rating the practice as "Not at all difficult". Observed infant behavior during the Intentional Introduction included such responses as "Eye opening", "Calm", and "Easier Transitioning to Care".

For the Intentional Exit observations, 100\% (10/10) of submissions indicated that nurses felt they were able to perform an Intentional Exit for the full amount of required time with $100 \%(10 / 10)$ of nurses rating the practice as "Not at all difficult". Observed infant behavior during the Intentional Exit 
included such responses as "Stable heart rate", "Decreased respiratory rate", and "Kept eyes open".

Responses on the Clinical Check-In assignments suggested that nurses were able to look at their infants and describe how intentional actions such as care introductions, care exits, and responding to stress signals seemed to result in their infants being better regulated. Nurses commented that the Intentional Introduction was, at times, "enough to calm the baby down without further intervention" or that the infant "opened his eyes but remained calm and his vital signs remained stable to his baseline". Nurses also expressed their internal reflection about Intentional Introductions in that, "It was a nice way to start the care and I believe it set the tone for the interaction" and "It's cool to see something as little as containing them for 5 seconds make such an impact on them rather than us just 'turning up the O's' or hurrying through the care just to finish it".

Participants' Clinical Mentorship Feedback Forms indicated that although all of the nurses started with a Care by Cues level of "3-Performs with competence," and could readily identify infant stress cues with antecedents, the majority of nurses (5/6) needed prompting by instructors to observe "shut-down" behavior and to describe methods that could be employed to facilitate the infant's recovery or return to a regulated state once the stress behaviors were observed. After the last phase, all nurses increased their Care by Cues level to 3.5 , with one nurse scoring a 4 .

Blinded review of videotaped caregiving sessions indicated that by Phase $\mathrm{C}$, all nurses demonstrated the ability to implement an Intentional Introduction and Intentional Exit. However, two nurses, who previously met criterion in Phase B, dropped the amount of their Intentional Introduction time below criterion level (see Figure 3). Analysis of the remaining structural components of nurses' caregiving (i.e., care time, number of caregiving tasks, and type of caregiving tasks) showed that the time that nurses spent per caregiving task increased for three nurses and decreased for two nurses from Video 1 to Video 2 (see Figure 4). However, the average time differential was negligible ( 31 seconds). It should be noted that one nurse's second video could not be analyzed for structural components as she chose to not further the caregiving process when the infant did not arouse from sleep.

\subsection{Nursing evaluation of the care by Cues process}

All participants reported (100\%) that they were confident in their understanding of Care by Cues and aware of the shortand long-term consequences of sleep deprivation and noxious experiences on neonatal development. All participants
(100\%) were also confident in their critical thinking skills and use of visual assessment to determine infant stability. They all (100\%) disagreed or strongly disagreed that performing care on a routine care schedule was best practice for NICU infants. The majority of participants (4/5) felt it was not best to complete tasks quickly to promote sleep. The identified barriers to Care by Cues implementation fell into two distinct themes: 1) unit mentality of schedules/policies and 2) lack of multi-disciplinary support.

\subsection{Infant behavioral and physiologic effects of the IOC segment of Care by Cues}

Behavioral data were recorded across sixteen caregiving interactions within a 48-hour period. Across all 16 interactions, two positive relationships were identified. First, a positive relationship was noted between the number of tasks completed and the frequency of dysregulation behavior during care (see Figure 5). Second, a positive relationship between the frequency of dysregulation behaviors and regulation behaviors during care (see Figure 6).

Three of the sixteen interactions were initiated with an IOC intervention. These two sets of data were compared with respect to length of the interaction; number of tasks completed during the interaction; frequency of dysregulation behaviors before, during and after the interaction; and frequency of regulation behaviors before, during and after the interaction. Additionally, relationships between tasks per minute during cares, time per task during cares, frequency of dysregulation behavior per minute during cares, frequency of regulation behavior per minute during cares, frequency of regulation behaviors per frequency of dysregulation behaviors during cares, frequency of dysregulation behavior after care and frequency of regulation behavior after care were calculated (see Table 3). The amount of time nurses spent with the infant during a care giving interaction increased by $34 \%$ when an IOC intervention was implemented. A similar 35\% increase in the time spent per task is noted with no significant change in the total number of tasks completed. IOC also resulted in a $35 \%$ decrease in the frequency of dysregulation behavior during care. More significantly, a nearly $70 \%$ decrease in the frequency of dysregulation behavior in the 10 minutes following completion of the caregiving interaction is noted when IOC is utilized (see Table 3). Behavior analytic line graphs (see Figure 7) demonstrate the impact of IOC on physiologic responses noted in the $\mathrm{ABAAB}$ intervention/withdrawal design. A generalized downward trend in heart rate and upward trend in oxygen saturations with increased stability in both measures is observed in the 10 minutes following care giving activities when the IOC intervention was utilized. 


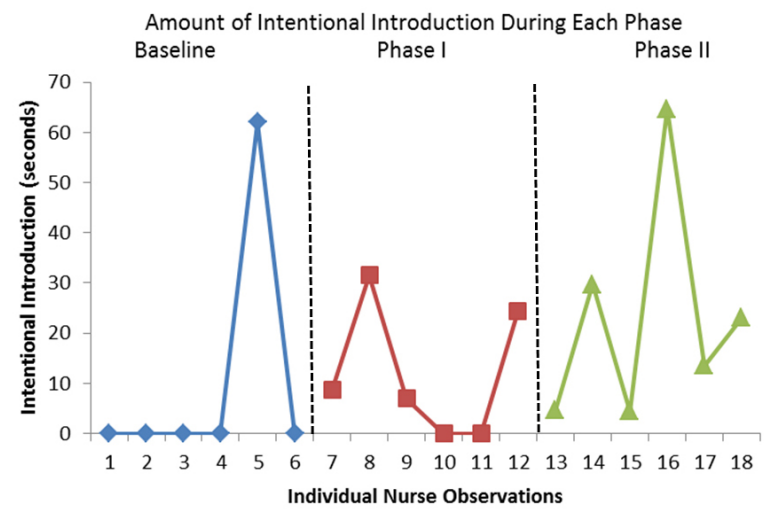

\begin{tabular}{|l|l|l|l|}
\hline Nurse & Baseline (seconds) & Phase I (seconds) & Phase II (seconds) \\
\hline 1 & 0 & 8.67 & 4.61 \\
\hline 2 & 0 & 31.57 & 29.71 \\
\hline 3 & 0 & 6.96 & 4.38 \\
\hline 4 & 0 & 0 & 64.7 \\
\hline 5 & 62 & 0 & 13.5 \\
\hline 6 & 0 & 24.45 & 23.05 \\
\hline
\end{tabular}

Figure 3. The length of Intentional Introduction increased between baseline and educational phases with all nurses demonstrating implementation of the minimum time requirement by Phase II. *Note, a video review of Nurse 5 at baseline indicated that the patient's age was 42 weeks corrected, unlike the remaining nurses' patients who were less than 28 weeks corrected

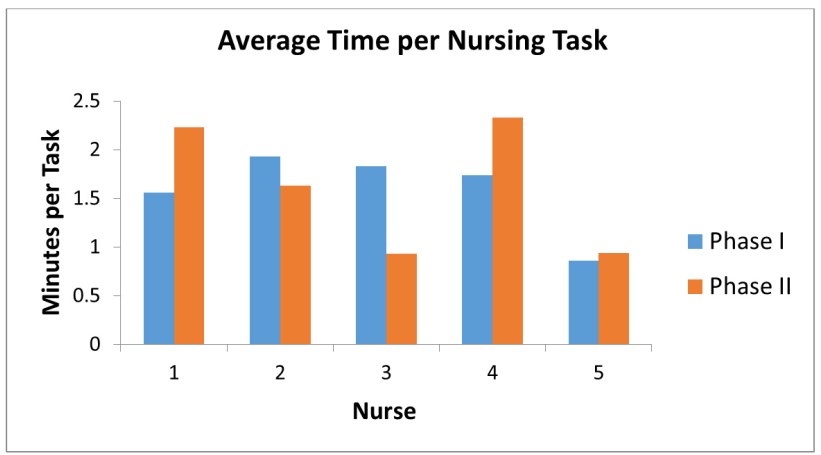

Figure 4. This graph depicts a comparison between educational phases in the amount of time nurses took per caregiving task. The intent of the Time per Task value is to serve as a proxy for intentionality in caregiving. Between phases, $3 / 5(60 \%)$ demonstrated some increase in task time

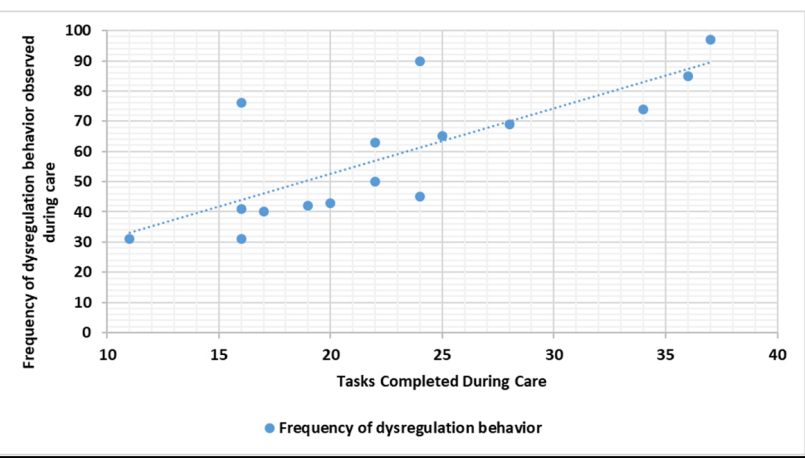

Figure 5. Frequency of infant dysregulation behavior per task during nurse caregiving interaction

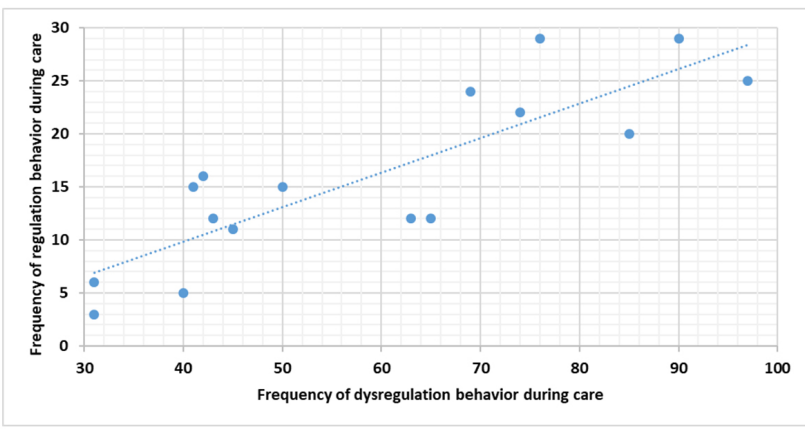

Figure 6. Frequency of infant dysregulation behavior per frequency of infant regulation behavior during care giving interaction

\section{Discussion}

The findings from our pilot study indicate that a systematic educational intervention, known as Care by Cues, has the potential to increase knowledge about infant behavior in nurses and to change clinical caregiving practice to promote increased infant regulation. The power of the educational approach may lie in the fact that, unlike traditional nursing education methodologies, the design included behavior analytic elements with natural positive reinforcement. This was necessitated by the intent of the intervention to not only increase nursing knowledge, but to also change standard nurse caregiving behavior.

The need to change nurse caregiving behavior is certainly not a new concern. Almost 40 years ago, Heidelise Als ${ }^{[6]}$ observed that preterm infants in the NICU exhibit distinct 
dysregulatory behaviors that are affected, in part, by how nurses interact during caregiving sessions. More recently, the trauma-informed care concept ${ }^{[4]}$ expanded on the need for higher quality caregiving interactions. Despite these efforts, preterm infants in the NICU, often still receive schedulebased caregiving. Recently, Zeiner, Storm, and Kopenhaver Doheny ${ }^{[15]}$ found that heart rate, respiratory rate, skin conductance frequency, and infant stress behaviors all significantly increased during nurse caregiving sessions with preterm infants.

In our study, we, too, noted dysregulatory effects of nurse caregiving on behavior, heart rate and oxygen saturation. However, we also demonstrated that incorporating small changes in nurses' perspectives of caregiving from completing tasks to more of a trauma-informed intentional healing ${ }^{[16]}$ process could change the behavior of how care was provided.
These small, intentional changes translated into improved infant regulation and moments of interpersonal experiences that nurses could readily describe (i.e., clinical check-in assignments). When nurses were taught to implement intentional introductions and intentional exits to their care, they were able to describe their patients as more regulated (i.e., "calm", "stable", and having an "easier transition to sleep" after care) and were able to describe caregiving as an interactional process. Additionally, after nurses used self-video analysis, they were able to read infant stress cues better and took more time with their patients. These findings coincide with the notion that traditional Continuing Medical Education formats and passive approaches have little effect on behavior change ${ }^{[17,18]}$ and that self-video analysis, when used as a supplement to traditional teaching methods, corresponds with greater clinical skill competency and more effective use of feedback. ${ }^{[19]}$

Table 3. Mean data and percent change for 11 parameters of care giving interactions with and without IOC. Boldface numbers highlight percent changes $>30 \%$

\begin{tabular}{llll}
\hline & IOC (Intervention) & No IOC (Baseline/Withdrawal) & \% Change \\
\hline Total time spent during care (min) & 23.333 & 17.308 & 34.815 \\
Number of tasks completed during cares & 23.333 & 22.846 & 2.132 \\
Number of tasks per minute during cares & 1.020 & 1.397 & -26.991 \\
Time spent per task during care (min) & 1.011 & 0.745 & 35.609 \\
Frequency of dysregulation behaviors during cares & 54 & 60 & -10 \\
Frequency of dysregulation behavior per minute during cares & 2.351 & 3.662 & -35.802 \\
Frequency of regulation behaviors during cares & 17 & 15.769 & 7.805 \\
Frequency of regulation behavior per minute during cares & 0.734 & 0.904 & -18.777 \\
Frequency of regulation behavior per frequency of dysregulation & 0.309 & 0.252 & 22.613 \\
behavior during care & & & -69.967 \\
Frequency of dysregulation behavior after care & 6.667 & 22 & -100 \\
Frequency of regulation behavior after care & 0 & 7.308 & \\
\hline
\end{tabular}

Finally, our in-depth single case study of behavioral and physiologic parameters showed a clear pattern of the potential regulatory effects of small changes in nurse caregiving. To our surprise, the intentional introduction had the most regulatory effect on the time period after care. When an intentional introduction was performed, a marked decrease in heart rate and increase in oxygen saturation after care, hints at an improved capacity for recovery after stressful events occurring during care. Heart rate and oxygen saturation are indicators of stress reactivity in preterm infants ${ }^{[15]}$ and infants under three months have demonstrated an elevation in cortisol levels during routine handling. ${ }^{[20]}$ Additionally, a positive relationship between regulatory and dysregulatory behavior frequency during care suggests that regulatory behaviors may, in fact, not represent regulation but the infant's attempt to return to a regulated state given the experience of stress or dysregulation. While it is positive that the infant is able to initiate these attempts to return to a regulated state, we must recognize the experience of stress that necessitates Published by Sciedu Press the use of these behavioral strategies to return to a positive, regulated state.

These findings open the thought process for future investigations into the effect of the Care by Cues intervention on cortisol reactivity. Future studies are also warranted in identifying small changes in practice during care that could potentially minimize physiologic and behavioral variability and to determine what aspects of Care by Cues have the most impact on regulation. The pilot study is limited in that it was implemented for a small group of nurses who were convenience sampled. Additionally, stress reactivity measures of heart rate and oxygen saturation indicated that the intentional introduction had a positive impact on infant stress regulation, but additional measures are needed for further justification. Finally, questions remain in how to a) logistically and efficiently implement the intervention on a larger scale and b) strengthen nurse conceptualization of the bi-directional nature of infant care in the NICU. 

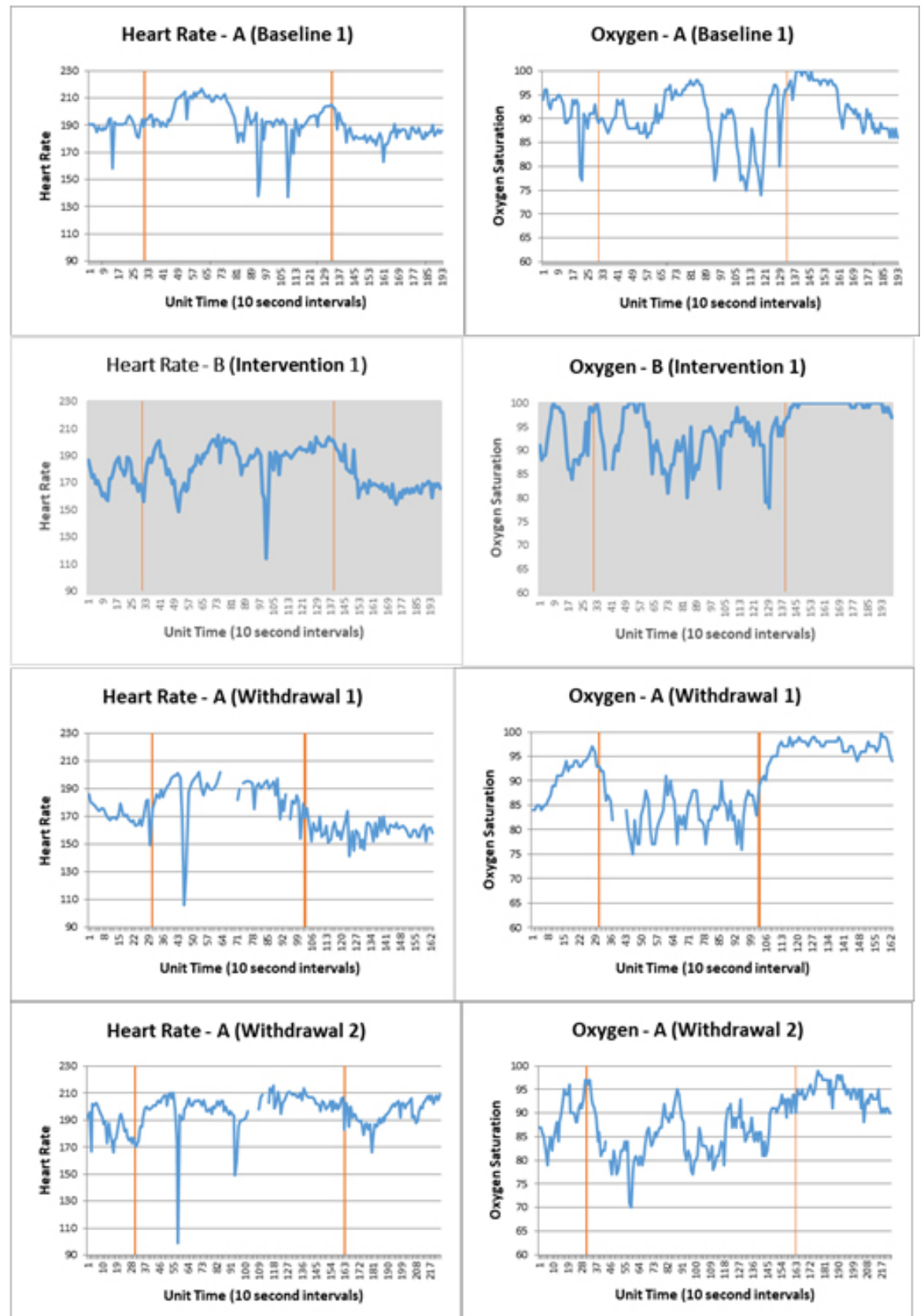

Oxygen - A (Withdrawal 2)
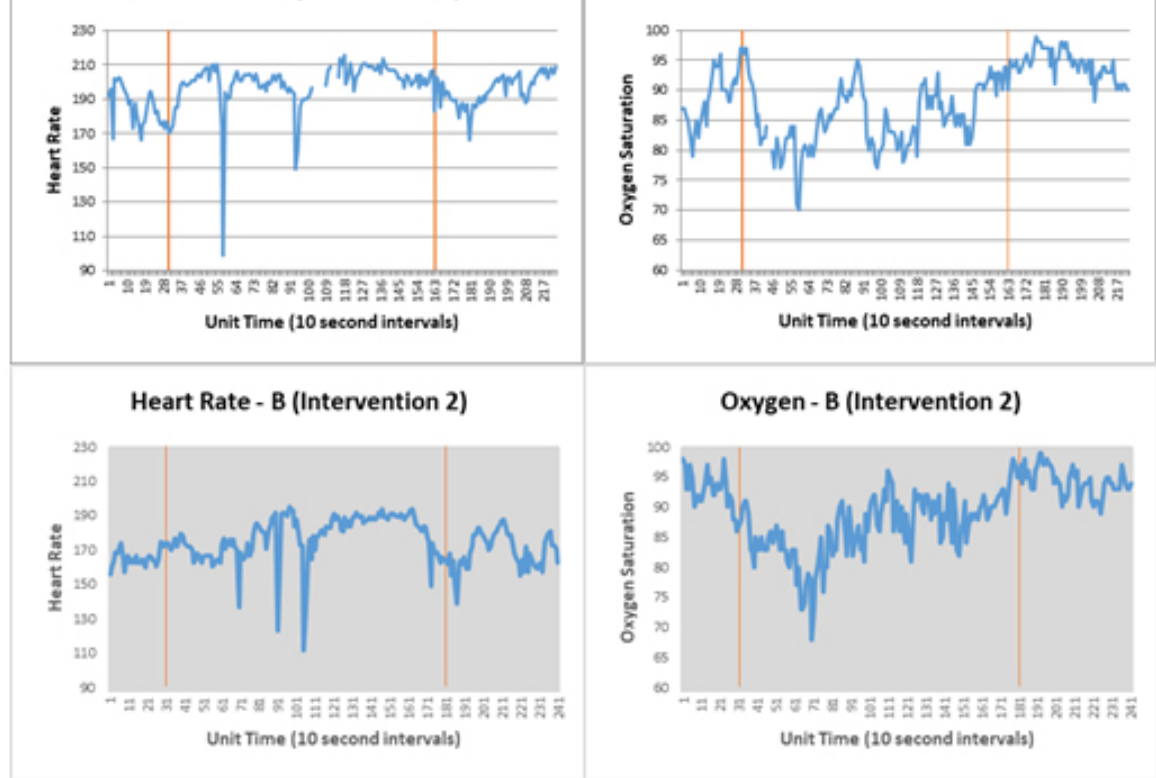

Figure 7. Behavioral line graphs representing 10 second intervals of heart rate (left) and oxygen saturation (right) readings for 5 minutes before a care giving event, during the entire event, and 10 minutes following the event. Vertical lines represent the start and end of the care giving event. Graphs represent ABAAB intervention/withdrawal design utilized with A representing baseline/withdrawal conditions and B representing intervention conditions. 


\section{Conclusion}

The Care by Cues pilot intervention succeeded in advancing nursing knowledge and changing practice behavior. There are also indications of positive impacts on infant regulation. However, caution should be taken in the study's limitations. Future studies are warranted in expanding the intervention to a larger population of nurses and more comprehensively measuring its effects on nursing practice and infant regulatory outcomes.

\section{CONFLICTS OF INTEREST Disclosure}

The authors declare that there is no conflict of interest.

\section{REFERENCES}

[1] McAnulty G, Duffy FH, Weisenfeld NI, et al. School age effects of the Newborn Individualized Developmental Care and Assessment Program for medically low-risk preterm infants: preliminary findings. J Clin Neonatol. 2012; 1(4): 184-194. PMid: 23951557 https://doi.org/10.4103/2249-4847.105982

[2] Newnham CA, Inder TE, Milgrom J. Measuring preterm cumulative stressors within the NICU: the Neonatal Infant Stressor Scale. Early Hum Dev. 2009; 85(9): 549-55. PMid: 19520525 https: //doi.org/1016/j.earlyhumdev.2009.05.002

[3] Als H, Duffy FH, McAnulty GB, et al. Early experience alters brain function. Pediatrics. 2004; 113(4): 846-57. PMid: 15060237

[4] Coughlin M. Trauma-Informed Care in the NICU. New York: Springer; 2017.

[5] Milette I, Martel MJ, Ribeiro da Silva M, et al. Guidelines for the institutional implementation of developmental neuroprotective care in the neonatal intensive care unit. Part A: background and rationale. A joint position statement from CANN, CAPWHNN NANN, and COINN. Can J Nurs Res. 2017. PMid: 28841058 https://doi.org/10.1177/0844562117706882

[6] Als H. Toward a synactive theory of development: promise for the assessment and support of infant individuality. Infant Ment Health J. 1982; 3(4): 229-43. https://doi.org/10.1002/1097-0355(19 8224) 3:4<229: : AID-IMHJ2280030405>3.0.CO;2-H

[7] Als H. A synactive model of neonatal behavioral organization: framework for the assessment of neurobehavioral development in the premature infant and for support of infants and parents in the neonatal intensive care environment. Phys Occup Ther Pediatr. 2009; 6(3-4): 3-53. https://doi.org/10.1080/J006v06n03_02

[8] Feldman R. The development of regulatory functions from birth to 5 years: insights from premature infants. Child Dev. 2009; 80(2): 544-561. Pmid: 19467010 https://doi.org/10.1111/j.1467 $-8624.2009 .01278 . x$

[9] Als H, McAnulty GB. The Newborn Individualized Developmental Care and Assessment Program (NIDCAP) with Kangaroo Mother Care (KMC): comprehensive care for preterm infants. Curr Womens Health Rev. 2011; 7(3): 288-301. PMid: 25473384 https: //doi.org/10.2174/157340411796355216
[10] Cooper JO, Heron TE, Heward WL. Applied Behavior Analysis Essex: Pearson; 2014.

[11] Coughlin M. Transformative Nursing in the NICU: Trauma Informed Age Appropriate Care. New York: Springer; 2014.

[12] Als H, Butler S, Kosta S, et al. The Assessment of Preterm Infants' Behavior (APIB): furthering the understanding and measurement of neurodevelopmental competence in preterm and full-term infants. Ment Retard Dev Disabil Res Rev. 2005; 11(1): 94-102. PMid:15856436 https ://doi.org/10.1002/mrdd. 20053

[13] Als H. Newborn Individualized Developmental Care and Assessment Program (NIDCAP) Guide. Boston: NIDCAP Federation International; 2015.

[14] Gast JR, Ledford JR. Single Case Research Methodology: Applications in Special Education and Behavioral Sciences (2nd Edition) New York: Taylor \& Francis; 2014.

[15] Zeiner V, Storm H, Kopenhaver Doheny K. Preterm infants' behavior and skin conductance responses to nurse handling in the NICU. J Matern Fetal Neonatal Med. 2017; 29(15): 2530-2535. PMid: 26440792 https://doi .org/10.3109/14767058.2015 1092959

[16] National Association of Neonatal Nurses (NANN). Age-Appropriate Care of the Premature and Critically Ill Hospitalized Infant: Guidelines for Practice. Glenview, Illinois: NANN; 2011.

[17] Davis D, Thomson O'Brien MA, Freemantle N, et al. Impact of formal continuing medical education. Do conferences, workshops, rounds, and other traditional continuing education activities change physician behavior or health care outcomes? JAMA. 1999; 282(9): 867-874. PMid: 10478694

[18] Grimshaw JM, Shirran L, Thomas R, et al. Changing provider behavior: an overview of systematic reviews of interventions. Med Care. 2001; 39(8): 112-45. PMid: 11583120

[19] Maloney S, Paynter S, Storr S, et al. Implementing student self-video of performance. Clin Teach. 2013; 10: 323-27. PMid: 24015739 https://doi.org/10.1111/tct.12027

[20] Laurent HK, Harold GT, Leve L, et al. Understanding the unfolding of stress regulation in infants. Dev Psychopathol. 2016; 28: 1431-1440. Pmid: 27020470 https://doi.org/10.1017/S0954579416000 171 